RAL-TR-99-032

May 1999

\title{
Can the trilinear Higgs self-coupling be measured at future linear colliders?
}

\author{
D.J. Miller' and S. Morettif \\ Rutherford Appleton Laboratory, \\ Chilton, Didcot, Oxon OX11 OQX, UK.
}

\begin{abstract}
We assess the possibility of measuring the trilinear self-coupling of the Standard Model Higgs boson at future electron-positron linear colliders with centre-of-mass energies between 500 and $1500 \mathrm{GeV}$. We consider rather light Higgs scalars, with mass below the $W^{+} W^{-}$threshold, produced in the double Higgs-strahlung channel $e^{+} e^{-} \rightarrow H H Z$ and decaying via the dominant mode $H \rightarrow b \bar{b}$. Assuming ideal vertex tagging of the heavy-quark jets and mass reconstruction of the $Z$ boson produced in association with the Higgs pair, we compare the yield of the above process to those of all irreducible electroweak and strong backgrounds proceeding through a $b \bar{b} b \bar{b} Z$ intermediate state. Total cross sections and differential spectra of phenomenological relevance to the selection and analysis of the signal are given and discussed.
\end{abstract}

\footnotetext{
${ }^{1}$ Email: D.J.Miller@rl.ac.uk
}

${ }^{2}$ Email: Moretti@v2.rl.ac.uk 


\section{Introduction}

In all probability a Higgs boson will be discovered at either LEP II, Run II of the Tevatron, or the Large Hadron Collider (LHC). It is then inevitable that the emphasis of Higgs physics will be turned away from discovery and instead will focus on the investigation of Higgs boson properties, such as its mass, width and branching ratios. Although much interesting Higgs phenomenology can be done at the LHC, many analyses are made infeasible by the rather messy nature of hadron colliders. Instead one must resort to the much cleaner environment of $e^{+} e^{-}$annihilations, for example, at the Linear Collider (LC) [1], where precision measurements at the $\mathrm{TeV}$ scale can be made.

One particularly interesting task to be carried out at future colliders is the reconstruction of the Higgs potential itself, possibly confirming, or denying, the mechanism of spontaneous electroweak symmetry breaking. This can be achieved by measuring the trilinear $\lambda_{H H H}$ and quadrilinear $\lambda_{H H H H}$ Higgs self-couplings, which can then be compared with the predictions of the Standard Model (SM), or indeed the Minimal Supersymmetric Standard Model (MSSM)円.

A measurement of the trilinear term, $\lambda_{H H H}$, is the first step in reconstructing the Higgs potential. At a future $e^{+} e^{-}$collider, the $\lambda_{H H H}$ coupling of the SM is accessible through double Higgs-strahlung off $Z$ bosons, in the process $e^{+} e^{-} \rightarrow H H Z$. This is the mechanism with which we will be concerned in this paper (for the MSSM see Ref. [8, 9]). The SM signal of the trilinear Higgs self-coupling has been thoroughly investigated in Ref. [9] (with its MSSM counterparts), and was found to be small but measurable for an intermediate mass Higgs boson, given a high integrated luminosity. In contrast, the quadrilinear vertex, $\lambda_{H H H H}$, is unmeasurable at the energy scale of the proposed LCs due to suppression by an additional power of the electromagnetic coupling constant.

However, in measuring $\lambda_{H H H}$, one must be sure that the already small signal can be distinguished from its backgrounds without being appreciably reduced. Here we will examine the $H \rightarrow b \bar{b}$ decay channel over the Higgs mass range $M_{H} \lesssim 140 \mathrm{GeV}$ and present kinematic cuts to aid its selection. The case of off- and on-shell $H \rightarrow W^{ \pm(*)} W^{\mp}$ decays for $M_{H} \gtrsim 140 \mathrm{GeV}$ is under examination elsewhere [10].

\footnotetext{
${ }^{3}$ In principle, the former coupling is amenable to investigation also at hadron and high energy photon colliders too, via double Higgs-strahlung off $W^{ \pm}$or $Z$ bosons [2, 3], $W^{+} W^{-}$or $Z Z$ fusion [3, 4, 5], gluon-gluon fusion [6] or $\gamma \gamma$ fusion [3, 田, 7].
} 
If one assumes very efficient tagging and high-purity sampling of $b$ quarks, the backgrounds to a $\lambda_{H н H}$ measurement from double Higgs events in the $4 b$ decay channel are primarily the 'irreducible' ones via $b \bar{b} b \bar{b} Z$ intermediate states [11], which can be separated into EW and QCD backgrounds. Furthermore, the double Higgs-strahlung process (see Fig. (1):

$$
e^{+} e^{-} \rightarrow H H Z \rightarrow b \bar{b} b \bar{b} Z
$$

contains diagrams proceeding via an $H H Z$ intermediate state but not dependent on $\lambda_{H H H}$ (graphs 1-3 in Fig. (4), as well as that sensitive to the trilinear Higgs self-coupling (graph 4 in Fig. (1). In addition, we also include four extra diagrams, which differ only in the exchange of the four-momenta and helicities of two identical $b$ quarks (or, equivalently, antiquarks) and a minus sign (due to Fermi-Dirac statistics pertinent to identical fermionic particles). However, the narrow width of the Higgs resonance ensures that the interference will be negligible and these extra diagrams could be included by symmetry.

The other two backgrounds proceed via purely EW interactions (see Figs. 20 2),

$$
e^{+} e^{-} \rightarrow \text { EW graphs } \rightarrow b \bar{b} b \bar{b} Z,
$$

and via QCD couplings as well (see Fig. 团),

$$
e^{+} e^{-} \rightarrow \text { QCD graphs } \rightarrow b \bar{b} b \bar{b} Z,
$$

and both contain no more than one intermediate Higgs boson. The EW background, process (2), is of $\mathcal{O}\left(\alpha_{e m}^{5}\right)$ away from resonances, but can, in principle, be problematic due to the presence of both $Z$ vectors and Higgs scalars yielding $b \bar{b}$ pairs. Finally, the QCD background, process (3), is of $\mathcal{O}\left(\alpha_{e m}^{3} \alpha_{s}^{2}\right)$ away from resonances. Here, although there are no heavy objects decaying to $b \bar{b}$ pairs, the production rate itself could give difficulties due to the presence of the strong coupling. As with process (1), one must include diagrams with the interchange of the two identical $b$ (anti)quarks also in the EW and QCD background processes. In contrast to the signal, here interference effects are sizable.

The plan of the paper is as follows. The next Section details the procedure adopted in computing the relevant scattering amplitudes. Sect. 3 displays our numerical results and contains our discussion. Finally, in the last Section, we summarize and conclude. 


\section{The matrix elements (MEs)}

The double Higgs-strahlung process (1) proceeds at lowest-order through the diagrams of Fig. 1, as explained in the Introduction. They are rather straightforward to calculate in the case of on-shell $H H Z$ production (see, e.g., Ref. [9] for an analytic expression of the $\mathrm{ME})$.

The EW background (2) derives from many graphs: 550 in total (again, considering the $b$ (anti)quark statistics). However, they can conveniently be grouped into different 'topologies': that is, collections of diagrams with identical (non-)resonant structure. We have isolated 23 of these, and displayed them in Figs. 2 and 3 , depending on whether one or zero Higgs intermediate states are involved, respectively. There are 214 graphs of the first kind and 336 of the second. This approach, of splitting the ME in (non-)resonant subprocesses, facilitates the integration over the phase space and further provides an insight into the fundamental dynamics. On the one hand, one can compute each of the topologies separately, with the appropriate mapping of variables, thus optimizing the accuracy of the numerical integration. On the other hand, one is able to assess the relative weight of the various subprocesses into the full scattering amplitude, by comparing the various integrals with each other. However, one should recall that the amplitudes squared associated to each of these topologies are in general non-gauge invariant. In fact, the latter is recovered

only when the various (non)-resonant terms are summed up. For reasons of space, we will not dwell in technicalities any further here, as a good guide to this technique can be found in Ref. [12]. (The resonant structure of the various subchannels ought to be self-evident in Figs. 20 2. )

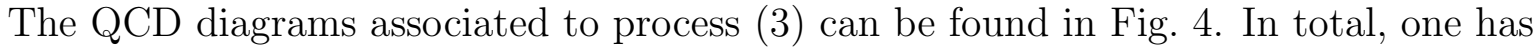
120 of these, with only five different (non-)resonant topologies. The integration in this case is much simpler than in the EW case and can in fact be carried out with percent accuracy directly over the full ME using standard multichannel Monte Carlo methods.

Non-zero interference effects exist between processes (14), (2) and (3). However, given the very narrow width of the Higgs boson (always below $20 \mathrm{MeV}$ over the mass range considered here), any interference with the signal can be safely neglected. Furthermore, we will see that the dominant subprocesses of the two backgrounds have very different topologies, so one also expects their interference to be negligible. Therefore, given that their calculation would be rather cumbersome, we do not consider them in our analysis. 
The large number of amplitudes can easily and efficiently be dealt with in the numerical evaluation if one resorts to helicity amplitudes. In doing so, we have adopted the HELAS subroutines [13]. The algorithm used to perform the multi-dimensional integrations was VEGAS [14].

Numerical inputs were as follows. The strong coupling constant $\alpha_{s}$ entering the QCD process (3) has been evaluated at two loops, with $N_{f}=5$ and $\Lambda_{\overline{\mathrm{MS}}}=160 \mathrm{MeV}$, at a scale equal to the collider CM energy, $\sqrt{s} \equiv E_{\mathrm{cm}}$. The EM coupling constant was $\alpha_{e m}=1 / 128$. The sine squared of the Weinberg angle was $\sin ^{2} \theta_{W}=0.232$. The fermionic (pole) masses were $m_{e}=0$ and $m_{b}=4.25 \mathrm{GeV}$. As for the gauge boson masses (and their widths), we have used: $M_{Z}=91.19 \mathrm{GeV}, \Gamma_{Z}=2.50 \mathrm{GeV}, M_{W} \equiv M_{Z} \cos \theta_{W} \approx 80 \mathrm{GeV}$ and $\Gamma_{W}=2.08 \mathrm{GeV}$.

Concerning the Higgs boson, we have spanned its mass $M_{H}$ over the range 100 to $150 \mathrm{GeV}$ and we have computed its width, $\Gamma_{H}$, by means of the program described in Ref. [15], which uses a running $b$ mass in evaluating the $H \rightarrow b \bar{b}$ decay fraction. Thus, for consistency, we have evolved the value of $m_{b}$ entering the $H b b$ Yukawa coupling of the $H \rightarrow b \bar{b}$ decay currents in the same way.

We have adopted as CM energies typical for the LC the values $E_{\mathrm{cm}}=500,1000$ and $1500 \mathrm{GeV}$.

Notice that, in the remainder of this paper, total and differential rates are those at the partonic level, as we identify jets with the partons from which they originate. In order to resolve the latter as four separate systems, we impose the following acceptance cuts: $E(b)>10 \mathrm{GeV}$ on the energy of each $b$ (anti)quark and $\cos (b, b)<0.95$ on the relative separation of all possible $2 b$ combinations. We further assume that $b$ jets are distinguishable from light-quark and gluon jets (e.g., by using $\mu$-vertex tagging techniques). However, no efficiency to tag the four $b$ quarks is included in our results. Also, the $Z$ boson is treated as on-shell and no branching ratio (BR) is applied to quantify its possible decays. In practise, in order to simplify the treatment of the final state, one may assume that the $Z$ boson decays leptonically (i.e., $Z \rightarrow \ell^{+} \ell^{-}$, with $\ell=e, \mu, \tau$ ) or hadronically into light quark jets (i.e., $Z \rightarrow q \bar{q}$, with $q \neq b$ ).

Finally, we have not included Initial State Radiation (ISR) [16] in our calculations. In fact, we would expect it to affect rather similarly the various processes (11)-(3). As we are basically interested in relative rates among the latter, we are confident that the salient features of our results are indifferent to the presence or not of photons radiated by the 
incoming electron-positron beams.

\section{Results}

The total cross sections for process (11), at the three CM energies considered here, can be

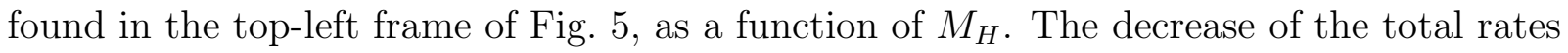
with increasing Higgs mass is mainly the effect of the BR of the decay channel $H \rightarrow b \bar{b}$, see, e.g., Fig. 1 of Ref. [15]. This mode is dominant and very close to 1 up to the opening of the off-shell $H \rightarrow W^{ \pm *} W^{\mp}$ decay channel, which occurs at $M_{H} \approx 140 \mathrm{GeV}$. In contrast, the production cross section for $e^{+} e^{-} \rightarrow H H Z$ is much less sensitive to $M_{H}$ [9]. In addition, because reaction (1) is an annihilation process proportional to $1 / s$, a larger $\mathrm{CM}$ energy tends to deplete the production rates, as long as $E_{\mathrm{cm}} \gg 2 M_{H}+M_{Z}$. When this is no longer true, e.g., at $500 \mathrm{GeV}$ and $M_{H} \gtrsim 140 \mathrm{GeV}$, phase space suppression can overturn the $1 / s$ propagator effects. This is evidenced by the crossing of the curves for 500 and $1000 \mathrm{GeV}$.

In practice, the maximum cross section for double Higgs-strahlung (1) is reached at energies $E_{\mathrm{cm}} \approx 2 M_{H}+M_{Z}+200 \mathrm{GeV}$ [9]. For Higgs masses in the lower part of the $M_{H}$ range considered here, e.g., $M_{H}=110 \mathrm{GeV}$ (where the bottom-antibottom channel is unrivaled by any other decay mode), this corresponds to $E_{\mathrm{cm}}=500 \mathrm{GeV}$. Furthermore, the sensitivity of the production rates of reaction (11) on $\lambda_{H H H}$ is higher at lower collider energies [9]. Thus, in order to illustrate the interplay between reactions (11)-(3), we will in the following focus on the case of a CM energy of $500 \mathrm{GeV}$, top-right corner of Fig. 5 , as an illustrative example. In fact, the discussion for the other two choices, $E_{\mathrm{cm}}=1000$ and $1500 \mathrm{GeV}$ (the two bottom plots of Fig. 5), would be rather similar, so we refrain from repeating it. (Also note that the signal-to-background $(S / B)$ ratio improves with increasing energy.)

The rise at $500 \mathrm{GeV}$ of the purely EW background (2) with the Higgs mass can be understood in the following terms. The dominant components of the EW process are those given by:

1. $e^{+} e^{-} \rightarrow Z Z Z \rightarrow b \bar{b} b \bar{b} Z$, first from the left in the second row of topologies in Fig. 3. That is, triple $Z$ production with no Higgs boson involved.

\footnotetext{
${ }^{4}$ We also neglect beamsstrahlung and Linac energy spread, by assuming a narrow beam design [16].
} 
2. $e^{+} e^{-} \rightarrow H Z Z \rightarrow b \bar{b} b \bar{b} Z$, first(first) from the left(right) in the fifth(fourth) row of topologies in Fig. 2 (also including the diagrams in which the on-shell $Z$ is connected to the electron-positron line). That is, single Higgs-strahlung production in association with an additional $Z$, with the Higgs decaying to $b \bar{b}$. The cross sections of these two channels are obviously identical.

3. $e^{+} e^{-} \rightarrow H Z \rightarrow Z^{*} Z^{*} Z \rightarrow b \bar{b} b \bar{b} Z$, first from the right in the third row of topologies in Fig. 2. That is, single Higgs-strahlung production with the Higgs decaying to $b \bar{b} b \bar{b}$ via two off-shell $Z^{*}$ bosons.

4. $e^{+} e^{-} \rightarrow Z H \rightarrow b \bar{b} Z^{*} Z \rightarrow b \bar{b} b \bar{b} Z$, first(first) from the right(left) in the first(second) row of topologies in Fig. 2. That is, two single Higgs-strahlung production channels with the Higgs decaying to $b \bar{b} Z$ via one off-shell $Z^{*}$ boson. Also the cross sections of these two channels are identical to each other, as in 2.

The production rates of 1.-4. as separate subprocesses can be found in the upper portion of Fig. 6. All other EW subprocesses are much smaller and rarely exceed $10^{-3}$ femtobarns, so we do not plot them here.

The QCD process (31) is dominated by $e^{+} e^{-} \rightarrow Z Z$ production with one of the two $Z$ bosons decaying hadronically into four $b$ jets. This subprocess corresponds to the topology in the middle of the first row of diagrams in Fig. 团. Notice that Higgs diagrams are involved in this process as well (bottom-right topology in the above figure). These correspond to single Higgs-strahlung production with the Higgs scalar subsequently decaying into $b \bar{b} b \bar{b}$ via an off-shell gluon. Their contribution is not negligible, owning to the large $Z H$ production rates, as can be seen in the lower portion of Fig. 6. The somewhat unexpected dependence of the latter upon $M_{H}$ (with a maximum at $130 \mathrm{GeV}$ ) is the result of the interplay between our acceptance cuts and phase space effects. The contribution of the other diagrams, which do not resonate, is typically one order of magnitude smaller than the $Z Z$ and $Z H$ mediated graphs, with the interferences even smaller (and generally negative).

One should note from Fig. 5 that the overall rates of the signal are quite small (also recall that we neglect tagging efficiency as well as the $Z$ decay rates), even at low Higgs masses where both the production and decay rates are largest. In fact, they are always below 0.2 femtobarns for all energies from 500 to $1500 \mathrm{GeV}$, although this can be doubled simply by polarizing the incoming electron and positron beams. Thus, as already recognised in Ref. 91, where on-shell production studies of process (10) were performed, 
luminosities of the order of one inverse attobarn need to be collected before statistically significant measurements of $\lambda_{H H H}$ can be performed. This emphasizes the need of high luminosity at any future LC.

We now proceed by looking at several differential spectra of reactions (11)-(3), in order to find suitable kinematic cuts which will enhance the $S / B$ ratio. The distributions in $E(b)$ and $\cos (b, b)$ leave little to exploit in separating signal from background after the acceptance cuts are made, especially with respect to the EW background. We turn then to other spectra, for example, invariant masses of $b$ (anti)quark systems. In this respect, we have plotted those of the following combinations:

$(\mathrm{a}, \mathrm{b})$ of $2 b$ systems, for the case in which the $b$ jets come from the same production vertex ('right' pairing) and the opposite case as well ('wrong' pairing);

(c) of $3 b$ systems, in which only two $b$ (anti)quarks have the same EM charge;

(d) of the $4 b$ system.

We denote the mass spectra of the systems (a)-(d) as $M_{R}(b b)$ and $M_{W}(b b)$ (where $R(W)$ signifies the right(wrong) combination), $M(b b b)$ and $M(b b b b)$, respectively. In the first three cases, there exists more than one combination of $b$ quarks. In such instances, we bin them all in the same distribution each with identical event weight. Further notice that the $2 b$ invariant masses that can be reconstructed experimentally are actually appropriately weighted superpositions of $M_{R}(b b)$ and $M_{W}(b b)$. In particular, if the $b$ charge tag is available, then it is roughly the sum of the two. If not, the latter is about twice as large as the former.

The invariant mass spectra can be found in Fig. 7, for the combination $E_{\mathrm{cm}}=500$ $\mathrm{GeV}$ and $M_{H}=110 \mathrm{GeV}$. Here, one can appreciate the narrow Higgs peak[ in the $M_{R}(b b)$ distribution, that can certainly be exploited in the signal selection, especially against the QCD background, which is rather flat in the vicinity of $M_{H}$. Apparently, this is no longer true for the EW process, as it also displays a resonance at $M_{H}$ (induced by the diagrams in Fig. 2 which carry an external on-shell current $H \rightarrow b \bar{b}$ ). However, events of the type (医) contain two $2 b$ invariant masses naturally peaking at $M_{H}$, whereas only one would

\footnotetext{
${ }^{5}$ Recall that for $M_{H}=110 \mathrm{GeV}$ one has $\Gamma_{H} \approx 3 \mathrm{MeV}$. The Higgs resonances in the top-left frame of Fig. 0 have been smeared out by incorporating a $5 \mathrm{GeV}$ bin width, emulating the finite efficiency of the detectors in determining energies and angles.
} 
appear in samples produced by process (2) (apart from accidental resonant mispairings). Thus, even in the case of the EW background one can achieve a significant noise reduction. Finally, requiring that none of the $2 b$ invariant masses reproduce a $Z$ boson will also be helpful in this respect, as evident from the $M_{R}(b b)$ spectrum of process (2). However, in this case, the invariant mass resolution of di-jet systems must be at least as good as the difference $\left(M_{H}-M_{Z}\right) / 2$, in order to resolve the $Z$ and $H$ peaks. Other mass distributions can be useful too in reducing the noise while keeping a substantial portion of the signal. Of some help are the $M(b b b)$ and $M(b b b b)$ spectra. In particular, notice that the minimum value of the latter is about $2 M_{H}$ for process (1), whereas for reaction (2) it is lower, typically around $2 M_{Z}$ or $M_{H}+M_{Z}$, as driven by the two dominant components of the EW background at low Higgs mass (i.e., subprocesses 1. and 2., respectively, see top of Fig. 6). The QCD background can stretch to $M(b b b b)$ values even further below the $2 M_{H}$ end point of the signal (the more the larger $M_{H}$ ), indeed showing a peak both at $M_{H}$ and $M_{Z}$, corresponding to the $H \rightarrow 4 b$ and $Z \rightarrow 4 b$ decays induced by the second and last topologies in Fig. 4 . As for the $M(b b b)$ spectrum, its shape is strongly related to that of the $4 b$ mass. In a sense, by excluding one of the four $b$ quarks from the mass reconstruction corresponds to smearing the $M(b b b b)$ distributions, so that the broad prominent peak at $M(b b b) \approx 90-100 \mathrm{GeV}$ in the case of the QCD process can be viewed as the superposition of the remains of the two narrow ones seen in $M(b b b b)$. For this very reason then, once a selection cut is imposed on one of the two masses, this is very likely to affect the other in a similar manner.

The spectacular differences seen in Fig. 7 between, on the one hand, process (1), and, on the other hand, reactions (2) and (3) (more in the former than in the latter), are a direct reflection of the rather different resonant structure of the various channels, that is, the form of the time-like propagators (i.e., $s$-channels) in the corresponding MEs. However, one should expect further kinematic differences, driven by the presence in the backgrounds of space-like propagators (i.e., $t, u$-channels), which are instead absent in the signal (see Figs. 1 (4). This is most evident in the properties of the four-quark hadronic system (d) recoiling against the $Z$ boson. As the internal dynamics of the four $b$ quarks is very different in each process (目)-(3), we also study the cases (a)-(c) separately.

One can appreciate the propagator effects by plotting, for example, the cosine of the polar angle (i.e., with the beam axes) of the four $b$ quark system (or, indeed, the real $Z$ ). See Fig. 8, where, again, $E_{\mathrm{cm}}=500 \mathrm{GeV}$ and $M_{H}=110 \mathrm{GeV}$. Notice that the 
backgrounds are much more forward peaked than the signal. This can be understood by recalling that the $\mathrm{QCD}$ events are mainly due to $e^{+} e^{-} \rightarrow Z Z$ production followed by the decay of one of the gauge bosons into four $b$ quarks. The $Z Z$ pair is produced via $t, u$-channel graphs, so the gauge bosons are preferentially directed forward and backward into the detector. In contrast, the signal (1) is always induced by $s$-channel graphs. The EW background (2) has a more complicated structure but is still sizably dominated by forward production. The behaviour of $\cos (b b b)$ and $\cos (b b)$ is very similar to that of the four-quark system. In practise, it is the strong boost of the $Z$ bosons produced forwards and backwards in processes (21)-(河), combined with the small value of $m_{b}$ (compared to the typical process scale, i.e., $E_{\mathrm{cm}}$ ), that produces a similar angular pattern for all multi- $b$

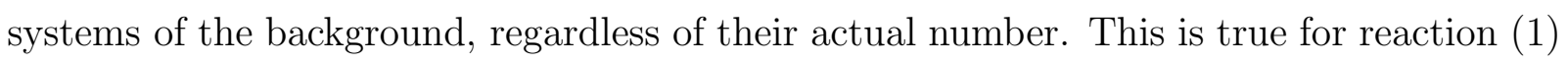
also. Therefore, all angular distributions displayed can boast strong (though correlated) discriminatory powers, allowing one to separate signal and backgrounds events efficiently.

An alternative possible means of disentangling the effects of the propagators is to resort to the differential distributions of the above systems (a)-(d) in transverse momentum, $p T$. These are plotted in Fig. 9, for the same $E_{\mathrm{cm}}$ and $M_{H}$ as the previous two figures. However, this kinematic variable proves not to be useful. In fact, the only discriminating distribution is the $p T$ for all four $b$ quarks (or equivalently the final state $Z$ boson), and this only singles out the QCD background, a large fraction of which populates the range beyond 180-200 GeV. Neither the signal nor the EW background do so and always look rather similar in their shape (even in the spectra involving $2 b$ quarks only, once these are appropriately combined together).

Notice that two of the $b$ quarks in the QCD background originate from gluon splitting and are therefore rather soft. Consequently, one expects the four $b$ quarks to be more planar, in the $4 b$ rest frame, for the QCD background than for the signal, where they are all the decay products of heavy bosons. We study this by plotting the thrust $T$ [17] and sphericity $S$ [18] distributions in Fig. 10. Indeed, such quantities could prove useful in reducing the QCD background but are harmless against the EW background.

Before proceeding to apply dedicated selection cuts, we remark that kinematic features similar to those displayed in Figs. 10 can also be seen at the other two values of CM energies considered here and for other Higgs masses. In fact, increasing the value of $M_{H}$ in those distributions mainly translates into a 'movable' resonant peak in $M_{R}(b b)$ as well as lower-end point in $M(b b b b)$ and into somewhat softer(harder) spectra in invariant 
mass(transverse momentum) than at the smaller $M_{H}$ value considered so far. Moreover, the $t, u$-channel dependence of the backgrounds, as opposed to the $s$-one of the signal, is more marked at higher $E_{\mathrm{cm}}$ values. Finally, for angular distributions, a larger Higgs mass does not remove the big differences seen between the three channels (11)-(3).

Therefore, in all generality, following our discussions of Figs. 7 10, and recalling the need to economize on the loss of signal because of its rather small production and decay rates, one can optimise the $S / B$ ratio by imposing the cuts:

$$
\begin{gathered}
\left|M(b b)-M_{H}\right|<5 \mathrm{GeV} \text { (on exactly two combinations of } 2 b \text { systems), } \\
\left|M(b b)-M_{Z}\right|>5 \mathrm{GeV} \text { (for all combinations of } 2 b \text { systems) } \\
M(b b b b)>2 M_{H}, \quad|\cos (2 b, 3 b, 4 b)|<0.75 .
\end{gathered}
$$

In enforcing these constraints, we assume no $b$ jet charge determination. Moreover, the reader should recall that the spectra of the four hadronic systems (a)-(d) are all correlated, in each of the quantities studied above, and so are the invariant masses, transverse momenta and polar angles among themselves.

The counterpart of Fig. 5 after the implementation of the above cuts is Fig. 11. The effect of the latter is a drastic reduction of both background rates (2) -(3), while maintaining a large portion of the original signal (1). Further notice how the imposition of the cuts (四) modifies the hierarchy of cross sections for process (11) with the CM energy, as now the largest rates occur at $E_{\mathrm{cm}}=1000 \mathrm{GeV}$ and the smallest at $E_{\mathrm{cm}}=500 \mathrm{GeV}$ (compare to Fig. 5).

The $S / B$ ratios turn out to be enormously large for not too heavy Higgs masses. For example, at $E_{\mathrm{cm}}=500(1000)[1500] \mathrm{GeV}$ and for $M_{H}=110 \mathrm{GeV}$, one gets $S / B=$ 25(60)[104], where $S$ corresponds to the rates of reaction (11) and $B$ refers to the sum of the cross sections for processes (2)-(3). The reduction of both backgrounds amounts to several order of magnitudes, particularly for the case of the strong process, whereas the loss of signal is much more contained. The acceptance of the latter is better at higher collider energies and lower Higgs masses. In fact, the poorest rate occurs for $E_{\mathrm{cm}}=500$ $\mathrm{GeV}$ at the upper end of the $M_{H}$ range, where more than $90 \%$ of the signal is sacrificed. We should however remark that the suppression of the backgrounds comes largely from the invariant mass cuts on $M_{b b}$ advocated in (幽. (In fact, they are crucial not only in selecting the $M_{H}$ resonance of the signal, but also in minimizing the rejection of the latter around 
$M_{Z}$ when mispairings occur: notice the shoulder at $90 \mathrm{GeV}$ of the $M_{W}(b b)$ spectrum of reaction (11)). The value we have adopted for the resolution is rather high, considering the large uncertainties normally associated with the experimental determination of jet angles and energies, though not unrealistic in view of the most recent studies [19]. The ability of the actual detectors in guaranteeing the performances foreseen at present is thus crucial for the feasibility of dedicate studies of double Higgs-strahlung events at the LC.

A related aspect is the efficiency of tagging the $b$ quarks necessarily present in the final state of reaction (11), particularly in the case in which the $Z$ boson decays hadronically. On the one hand, given the high production rate of six jet events from QCD 20 and multiple gauge boson resonances [21] in light quark and gluon jets, it is desirable to resort to heavy flavour identification in hadronic samples. On the other hand, the poor statistics of the $H H Z$ signal requires a judicious approach in order not to deplete the latter below detection level. According to recent studies [22], the two instances can be combined successfully, as efficiencies for tagging $b \bar{b}$ pairs produced in Higgs decays were computed to be as large as $\epsilon_{b \bar{b}} \approx 90 \%$, with mis-identification probabilities of light(charmed) quarks as low as $\epsilon_{q \bar{q}(c \bar{c})} \approx 0.3(4) \%$ (and negligible for gluons). If such a projection for the LC detectors proves to be true, then even the requirement of tagging exactly four $b$ quarks in double-Higgs events of the type (四) might be statistically feasible, thus suppressing the reducible backgrounds to really marginal levels [11].

One must also bear in mind that experimental considerations, such as the performances of detectors, the fragmentation/hadronization dynamics and a realistic treatment of the $Z$ boson decays, are also important when determining what cuts should be made. Such considerations are beyond the scope of this paper, and are under study elsewhere [11].

Finally, the number of signal and backgrounds events seen per inverse attobarn of luminosity at $E_{\mathrm{cm}}=500,1000$, and $1500 \mathrm{GeV}$, with $M_{H}=110 \mathrm{GeV}$, can be seen in Tab. 1. Of course, one could relax one or more of the constraints we have adopted to try to improve the signal rates without letting the backgrounds become unmanageably large. For example, by removing the cuts on $\cos (b b)$ and $\cos (b b b)$ one can enhance the signal rates by about a factor of two. However, the EW background would also increase by a comparable amount and the QCD rate would do by a somewhat larger factor, of at least three/four. Kinematic fits can also help in improving the $S / B$ ratios [11]. 


\section{Summary}

In conclusion, the overwhelming irreducible background from EW and QCD processes of the type $e^{+} e^{-} \rightarrow b \bar{b} b \bar{b} Z$ to double Higgs production in association with $Z$ bosons and decay in the channel $H \rightarrow b \bar{b}$, i.e., $e^{+} e^{-} \rightarrow H H Z \rightarrow b \bar{b} b \bar{b} Z$, should easily be suppressed down to manageable levels by simple kinematics cuts: e.g., in invariant masses and polar angles.

The number of signal events is generally rather low, but will be observable at the LC given the following 'mandatory conditions' (some of which have already been outlined in Ref. [11):

- very high luminosity;

- excellent $b$ tagging performances;

- high di-jet resolution.

The requirement advocated in Ref. [11] of a good forward acceptance for jets may also be added to the above list, as we have explicitly verified (though not shown) that single jet directions can stretch in the double Higgs-strahlung process up to about 20 degrees in polar angle. Finally, beam polarization can also be invoked to increase the signal-to-background rates [9].

\section{Acknowledgments}

We thank Peter Zerwas for suggesting the subject of this research and for useful discussions. DJM would also like to thank DESY for hospitality while part of this research was carried out. Financial support from the UK-PPARC is gratefully acknowledged by both authors.

\section{References}

[1] Proceedings of the Workshop $e^{+} e^{-}$Collisions at $500 \mathrm{GeV}$. The Physics Potential, Munich, Annecy, Hamburg, 3-4 February 1991, ed. P.M. Zerwas, DESY 92-123A/B, August 1992, DESY 93-123C, December 1993.

E. Accomando et al., Phys. Rep. Vol. 299, No. 1 (1998) 1. 
[2] G. Gounaris, D. Schildknecht and F. Renard, Phys. Lett. B83 (1979) 191; Erratum, ibidem B89 (1980) 437;

V. Barger, T. Han and R.J.N. Phillips, Phys. Rev. D38 (1988) 2766.

[3] V.A. Ilyin, A.E. Pukhov, Y. Kurihara, Y. Shimizu and T. Kaneko, Phys. Rev. D54 (1996) 6717.

[4] F. Boudjema and E. Chopin, Z. Phys. C71 (1996) 431.

[5] V. Barger and T. Han, Mod. Phys. Lett. A5 (1990) 667;

A. Dobrovolskaya and V. Novikov, Z. Phys. C52 (1991) 427;

D.A. Dicus, K.J. Kallianpur and S.S.D. Willenbrock, Phys. Lett. B200 (1998) 187;

A. Abbasabadi, W.W. Repko, D.A. Dicus and R. Vega, Phys. Rev. D38 (1998) 2770;

Phys. Lett. B213 (1998) 386.

[6] E.W.N. Glover and J.J. van der Bij, Nucl. Phys. B309 (1988) 282;

T. Plehn, M. Spira and P.M. Zerwas, Nucl. Phys. B479 (1996) 46; Erratum, ibidem B531 (1998) 655;

S. Dawson, S. Dittmaier and M. Spira, Phys. Rev. D58 (1998) 115012.

[7] G. Jikia, Nucl. Phys. B412 (1994) 57.

[8] A. Djouadi, H.E. Haber and P.M. Zerwas, Phys. Lett. B375 (1996) 203;

P. Osland and P.N. Pandita, Phys. Rev. D59 (1999) 055013; preprint BERGEN-199901, February 1999, hep-ph/9902270;

P. Osland, preprint ISSN 0803-2696, March 1999, hep-ph/9903301.

[9] A. Djouadi, W. Kilian, M. Muhlleitner and P.M. Zerwas, preprint DESY 99/001, TTP99-02, PM/99-01, March 1999, hep-ph/9903229; contribution to the XXIX International Conference on High Energy Physics, Vancouver 1998, Heidelberg Report HD-THEP 98-29.

[10] D.J. Miller and S. Moretti, in preparation.

[11] P. Lutz, talk delivered at the ECFA/DESY Workshop on "Physics and Detectors for a Linear Collider", Oxford, UK, March 20-23, 1999. 
[12] A. Ballestrero, E. Maina and S. Moretti, Phys. Lett. B335 (1994) 460;

S. Moretti, Phys. Rev. D50 (1995) 6316; Z. Phys. C73 (1997) 653;

S. Moretti and K. Odagiri, Eur. Phys. J. C1 (1998) 633.

[13] H. Murayama, I. Watanabe and K. Hagiwara, HELAS: HELicity Amplitude Subroutines for Feynman Diagram Evaluations, KEK Report 91-11, January 1992.

[14] G.P. Lepage, Jour. Comp. Phys. 27 (1978) 192.

[15] Z. Kunszt, S. Moretti and W.J. Stirling, Z. Phys. C74 (1997) 479.

[16] T. Barklow, P. Chen and W. Kozanecki, in Ref. [1], part A.

[17] E. Fahri, Phys. Rev. Lett. 39 (1977) 1587.

[18] J.D. Bjorken and S.J. Brodsky, Phys. Rev. D1 (1970) 1416.

[19] F. Richard, private communication.

[20] S. Moretti, Phys. Lett. B420 (1998) 367; Nucl. Phys. B544 (1999) 289.

[21] A. Aeppli et. al., in Ref. [1], part A;

V. Barger and T. Han, Phys. Lett. B212 (1988) 117;

V. Barger, T. Han and R.J.N. Phillips, Phys. Rev. D39 (1889) 146;

A. Tofighi-Niaki and J.F. Gunion, Phys. Rev. D39 (1989) 720.

[22] G. Borissov, talk delivered at the ECFA/DESY Workshop on "Physics and Detectors for a Linear Collider", Oxford, UK, March 20-23, 1999;

M. Battaglia, ibidem. 


\begin{tabular}{|c||c|c|c|}
\hline \multicolumn{1}{|c||}{} & \multicolumn{3}{c|}{ Number of Events per $a b^{-1}$ after selection cuts } \\
\cline { 2 - 4 } & $E_{\mathrm{cm}}=500 \mathrm{GeV}$ & $E_{\mathrm{cm}}=1000 \mathrm{GeV}$ & $E_{\mathrm{cm}}=1500 \mathrm{GeV}$ \\
\hline \hline signal & 26 & 40 & 34 \\
\hline Electroweak & 1.0 & 0.6 & 0.3 \\
\hline QCD & 0.032 & 0.026 & 0.016 \\
\hline
\end{tabular}

Table 1: The number of events for signal and backgrounds per inverse attobarn of luminosity after selection cuts for centre-of-mass energies of 500, 1000 and $1500 \mathrm{GeV}$, a Higgs mass of $110 \mathrm{GeV}$, and with polarized electron and positron beams.
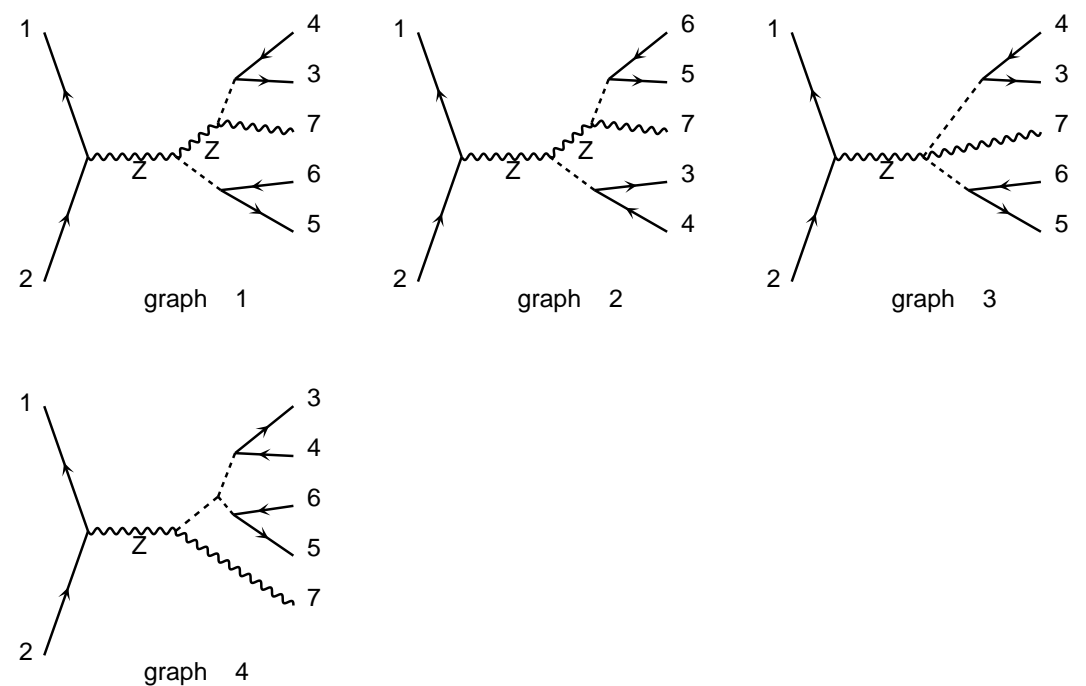

Figure 1: Diagrams contributing at lowest-order to $e_{1}^{+} e_{2}^{-} \rightarrow b_{3} \bar{b}_{4} b_{5} \bar{b}_{6} Z_{7}$ via purely EW interactions containing two Higgs bosons in intermediate states. An internal wavy line corresponds to a $Z$ boson (labeled as $\mathrm{Z}$ ). The total number of actual diagrams is 4 . Finally, diagrams which differ from those above only in the exchange $3 \leftrightarrow 5$ (or, equivalently, $4 \leftrightarrow 6$ ) must also be considered, preceded by a minus sign. 

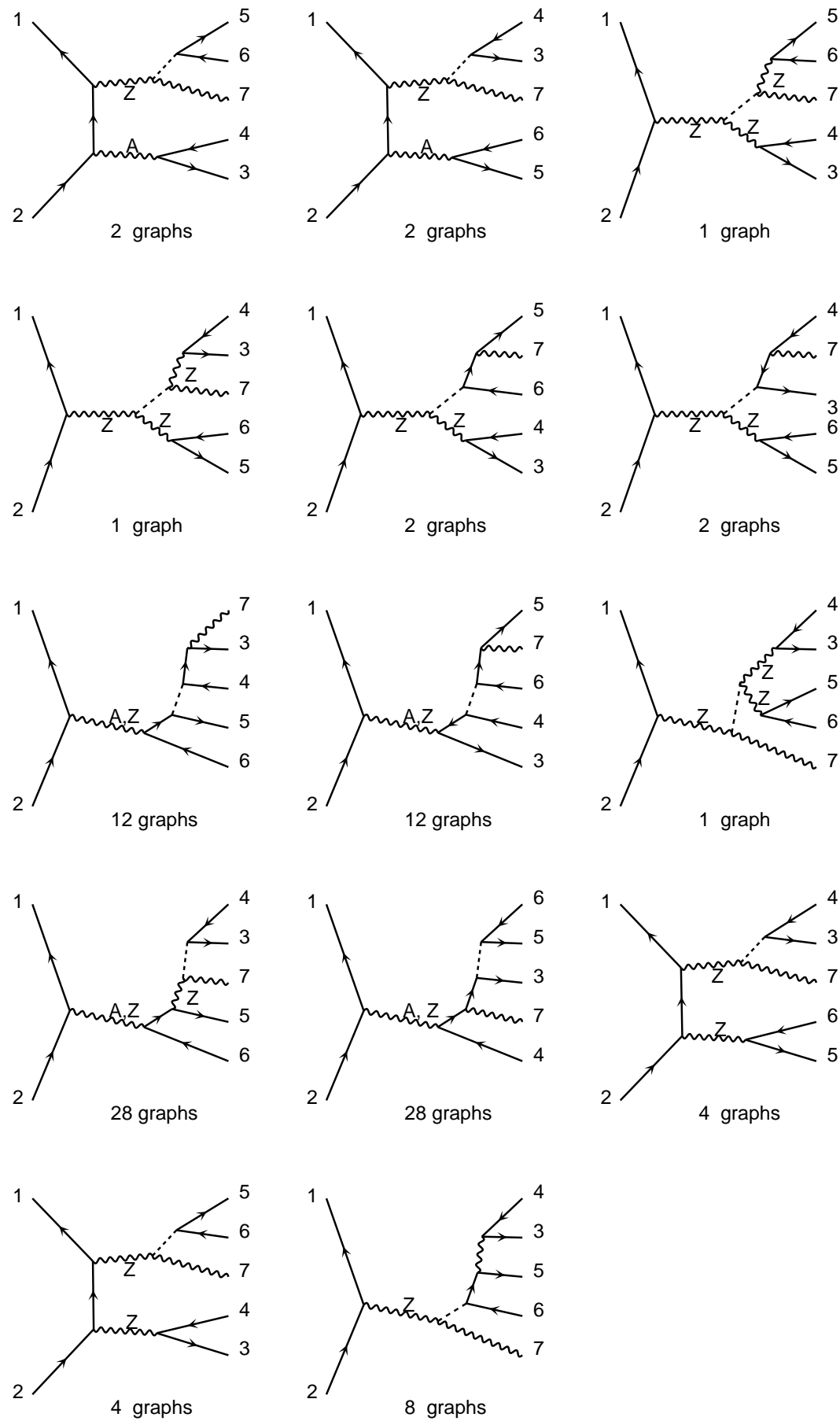

Figure 2: Topologies contributing at lowest-order to $e_{1}^{+} e_{2}^{-} \rightarrow b_{3} \bar{b}_{4} b_{5} \bar{b}_{6} Z_{7}$ via purely EW interactions containing only one Higgs boson in intermediate states. An internal wavy line corresponds to a $\gamma$ or a $Z$ (labeled as A and Z, respectively), as appropriate. The total number of actual diagrams is 107. Finally, diagrams which differ from those above only in the exchange $3 \leftrightarrow 5$ (or, equivalently, $4 \leftrightarrow 6$ ) must also be considered, preceded by a minus sign. 

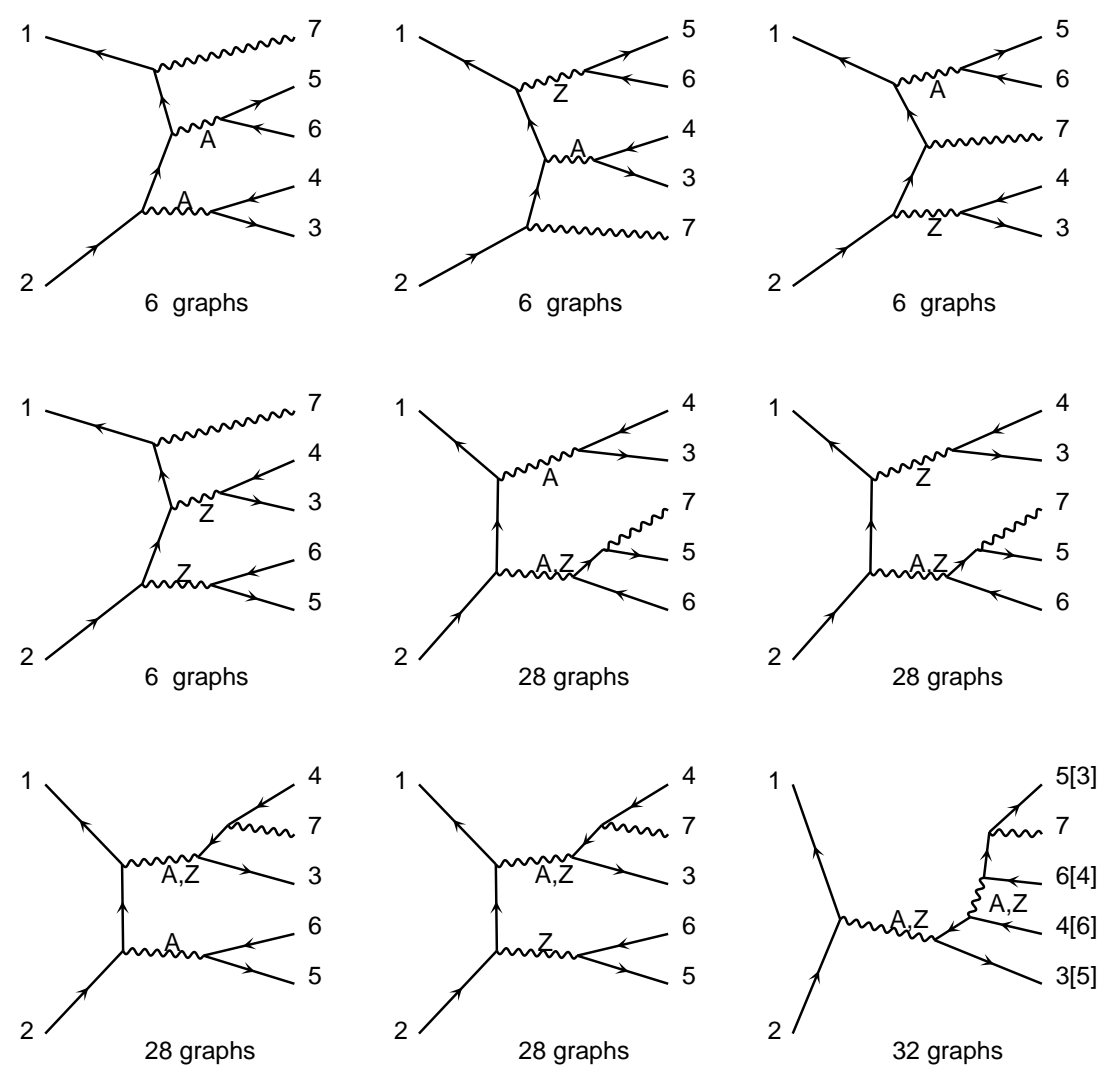

Figure 3: Topologies contributing at lowest-order to $e_{1}^{+} e_{2}^{-} \rightarrow b_{3} \bar{b}_{4} b_{5} \bar{b}_{6} Z_{7}$ via purely EW interactions containing no Higgs bosons in intermediate states. An internal wavy line corresponds to a $\gamma$ or a $Z$ (labeled as A and Z, respectively), as appropriate. The total number of actual diagrams is 168 . Finally, diagrams which differ from those above only in the exchange $3 \leftrightarrow 5$ (or, equivalently, $4 \leftrightarrow 6$ ) must also be considered, preceded by a minus sign. 

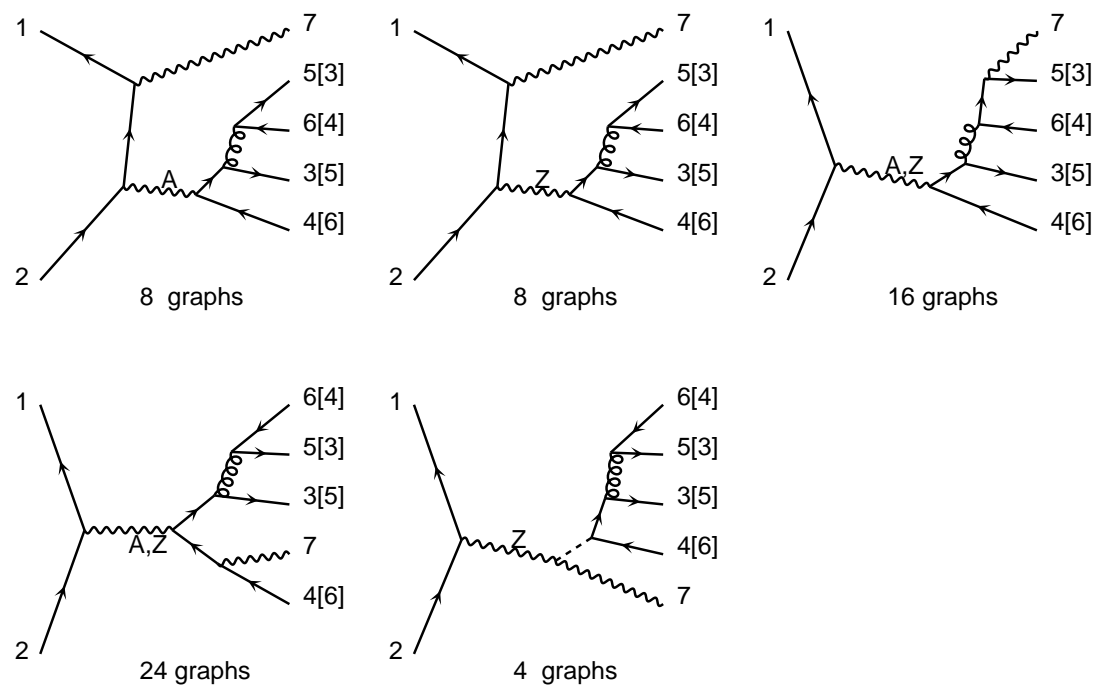

Figure 4: Topologies contributing at lowest-order to $e_{1}^{+} e_{2}^{-} \rightarrow b_{3} \bar{b}_{4} b_{5} \bar{b}_{6} Z_{7}$ via QCD interactions containing one gluon in intermediate states. An internal wavy line corresponds to a $\gamma$ or a $Z$ (labeled as A and Z, respectively), as appropriate, whereas a helical one refers to a $g$. The total number of actual diagrams is 60 . Finally, diagrams which differ from those above only in the exchange $3 \leftrightarrow 5$ (or, equivalently, $4 \leftrightarrow 6$ ) must also be considered, preceded by a minus sign. 

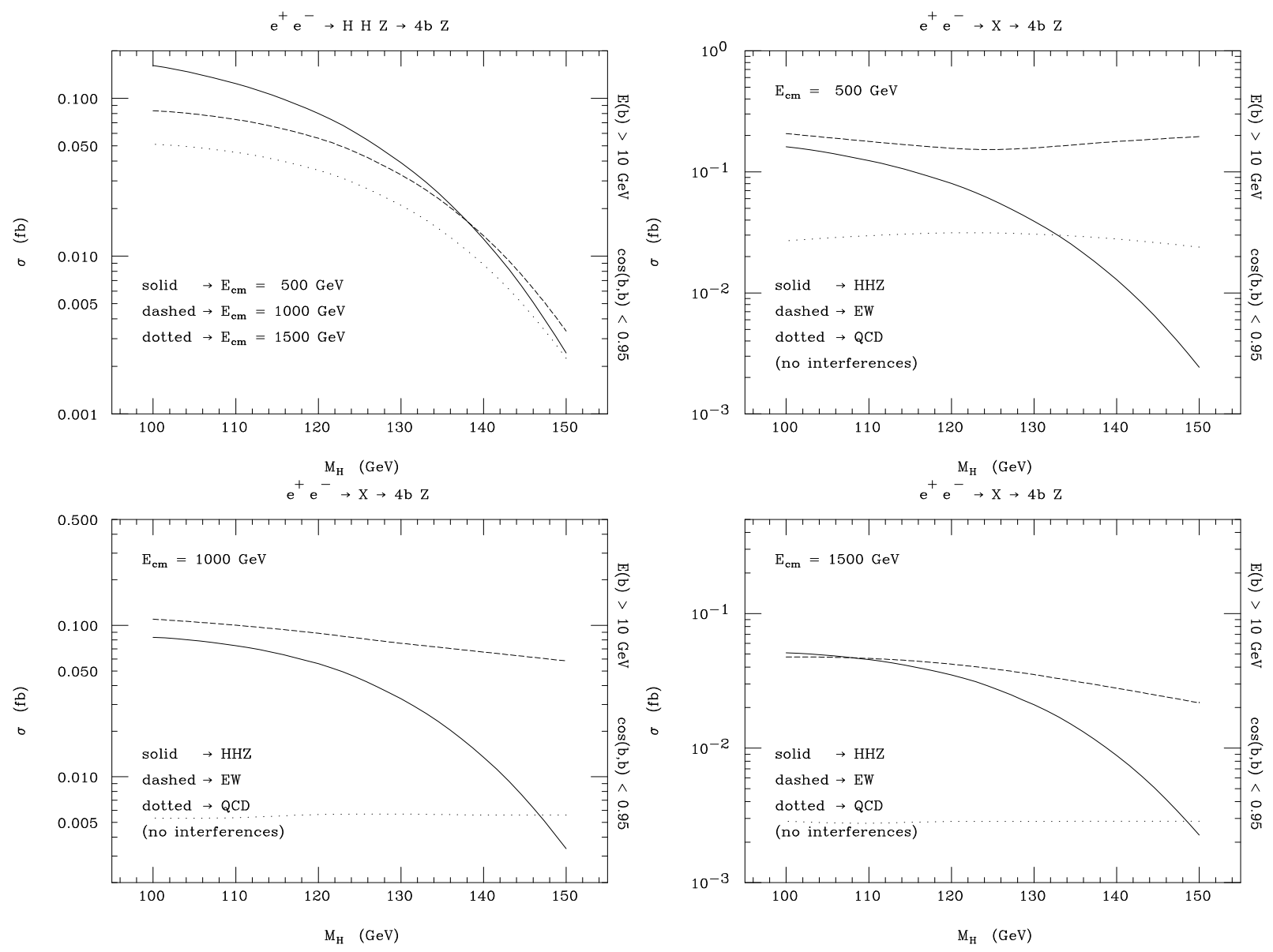

Figure 5: Top-left: cross sections in femtobarns for the signal at three different collider energies: 500, 1000 and $1500 \mathrm{GeV}$. Top-right(Bottom-left)[Bottom-right]: cross sections in femtobarns for the signal versus the EW and QCD backgrounds at 500(1000)[1500] GeV. Our acceptance cuts in energy and separation of the four $b$ quarks have been implemented. 

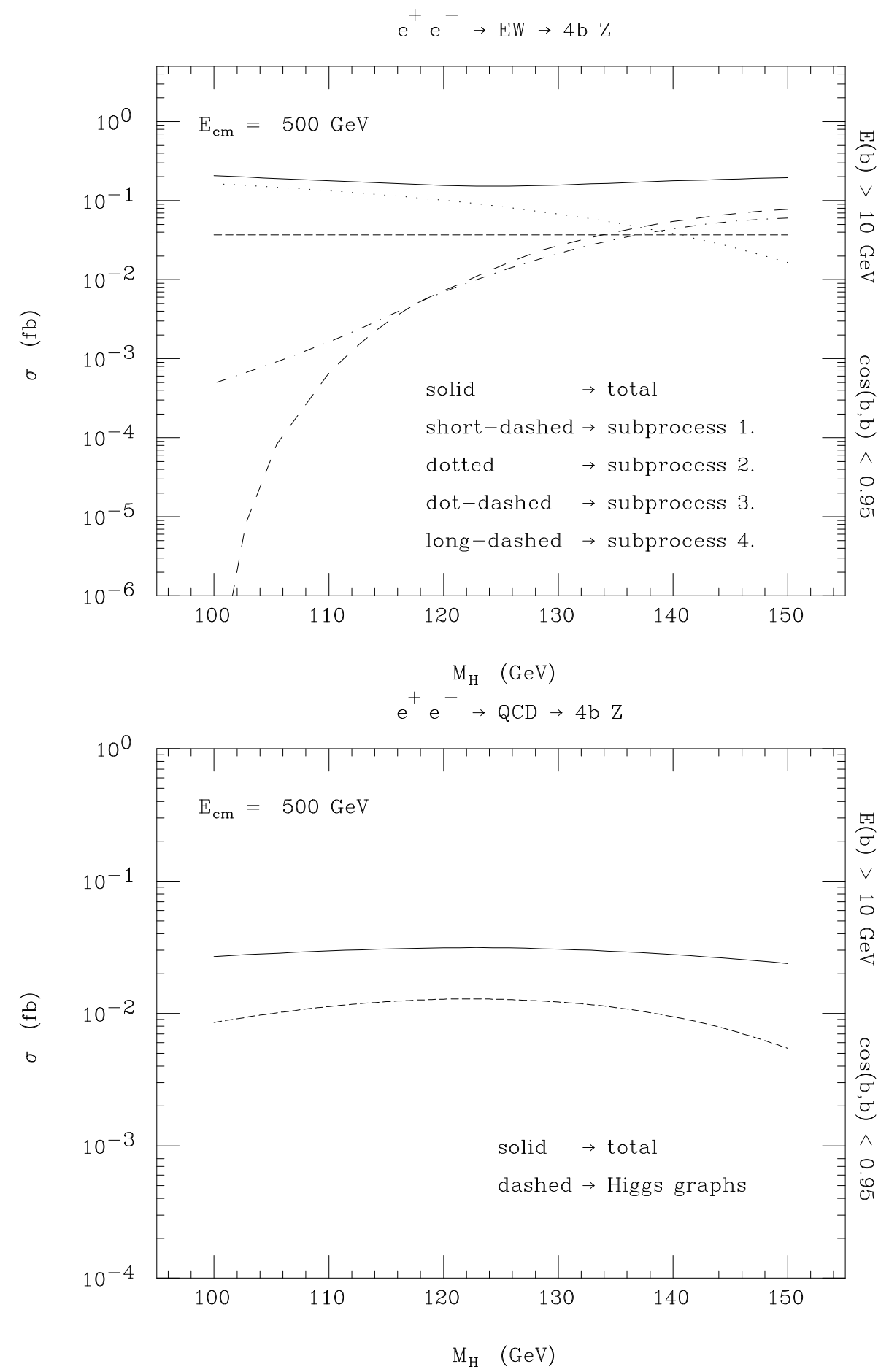

Figure 6: Top: cross sections in femtobarns for the four dominant components (see the text) of the purely EW background. Bottom: cross sections in femtobarns for the total and Higgs components (see the text) of the QCD background. The CM energy is $500 \mathrm{GeV}$. Our acceptance cuts in energy and separation of the four $b$ quarks have been implemented. 


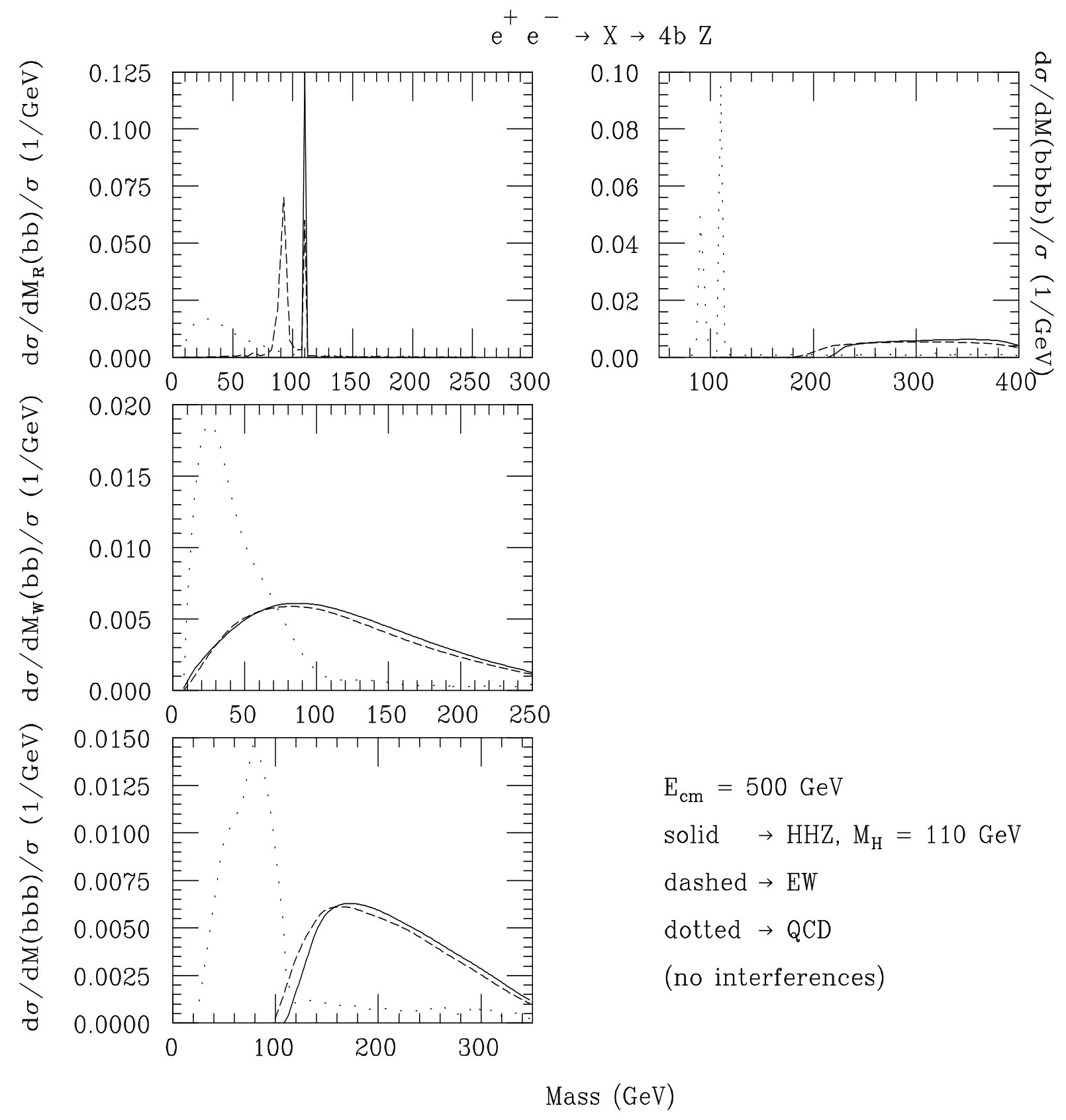

Figure 7: Differential distributions in invariant mass of multi-jet systems containing one, two, three and four $b$ (anti)quarks. The CM energy is $500 \mathrm{GeV}$ and the Higgs mass $110 \mathrm{GeV}$. Our acceptance cuts in energy and separation of the four $b$ quarks have been implemented. 


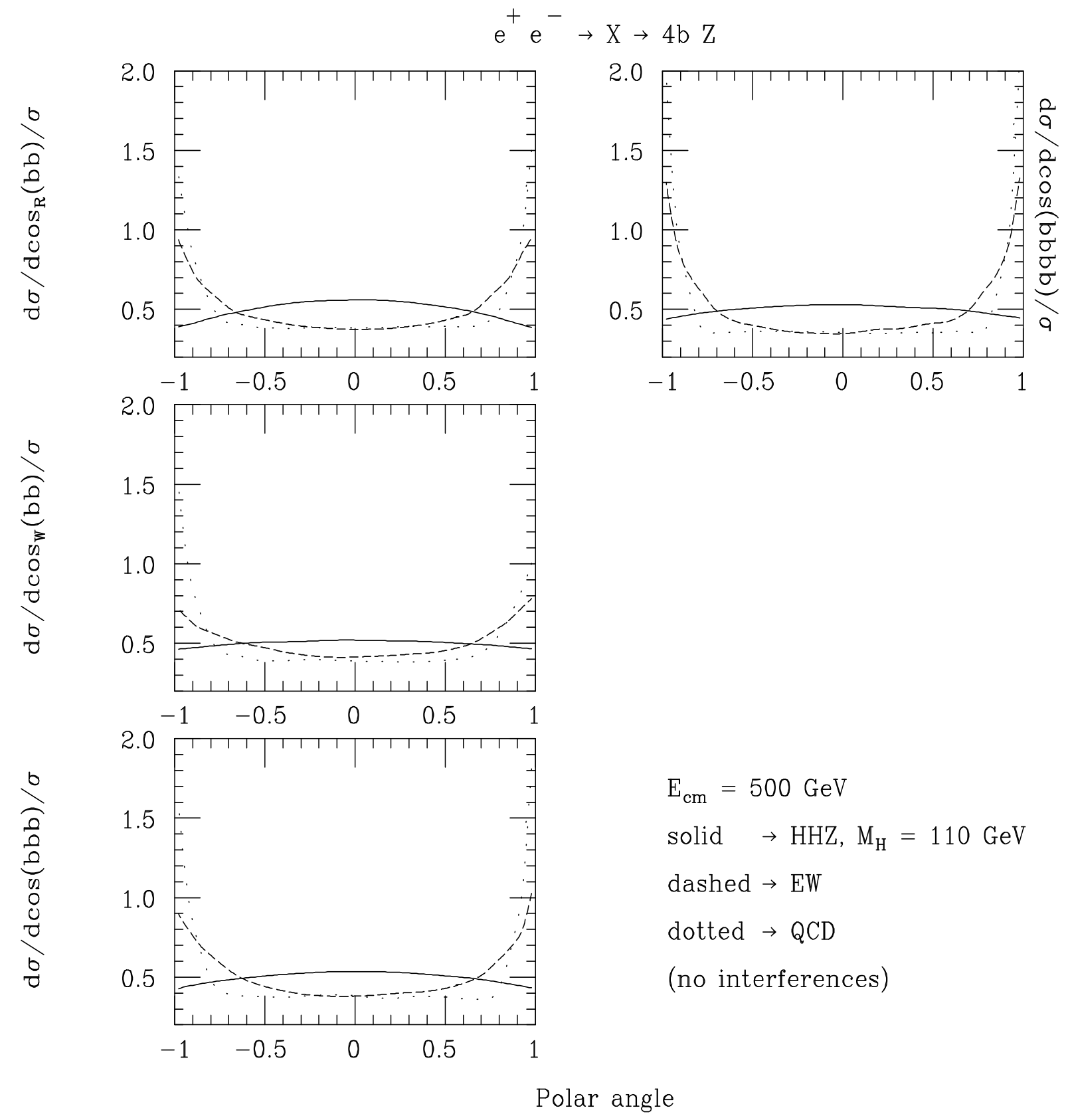

Figure 8: Differential distributions in (cosine of the) polar angle of multi-jet systems containing one, two, three and four $b$ (anti)quarks. (The spectrum of the $4 b$ system is identical to that of the $Z$ boson.) The CM energy is $500 \mathrm{GeV}$ and the Higgs mass $110 \mathrm{GeV}$. Our acceptance cuts in energy and separation of the four $b$ quarks have been implemented. 


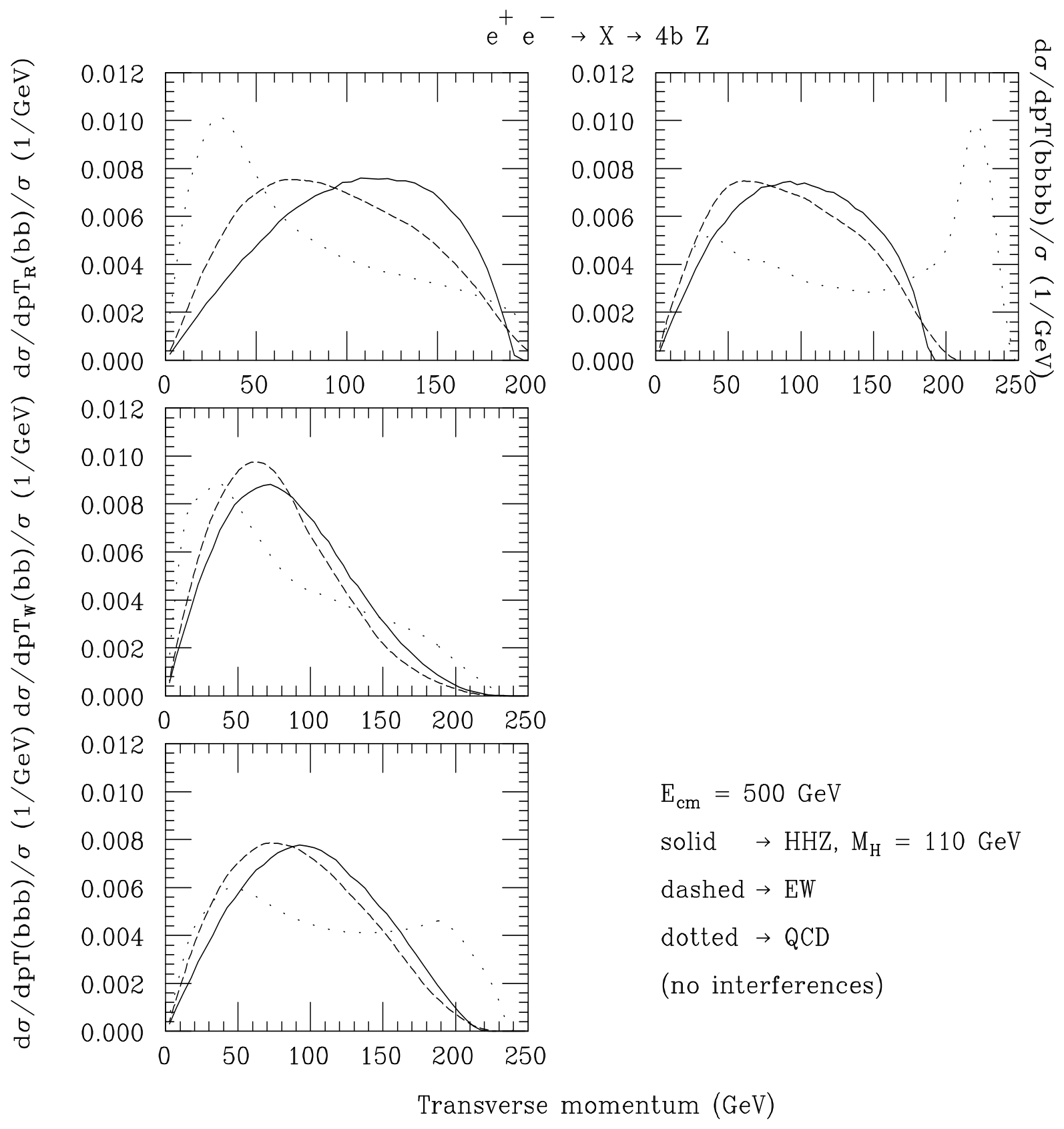

Figure 9: Differential distributions in transverse momentum of multi-jet systems containing one, two, three and four $b$ (anti)quarks. (The spectrum of the $4 b$ system is identical to that of the $Z$ boson.) The CM energy is $500 \mathrm{GeV}$ and the Higgs mass $110 \mathrm{GeV}$. Our acceptance cuts in energy and separation of the four $b$ quarks have been implemented. 

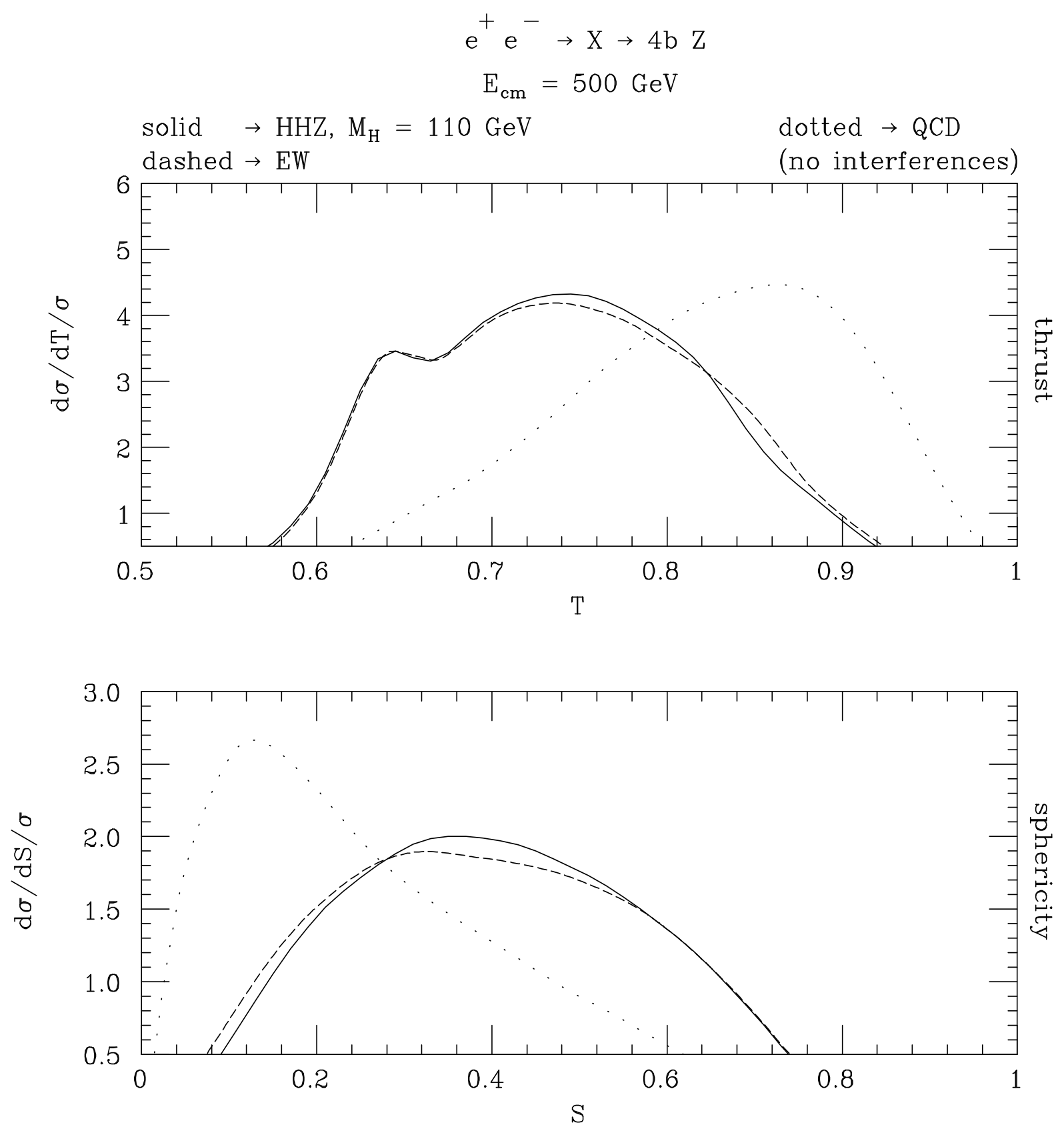

Figure 10: Differential distributions in thrust and sphericity. The CM energy is $500 \mathrm{GeV}$ and the Higgs mass $110 \mathrm{GeV}$. Our acceptance cuts in energy and separation of the four $b$ quarks have been implemented. 

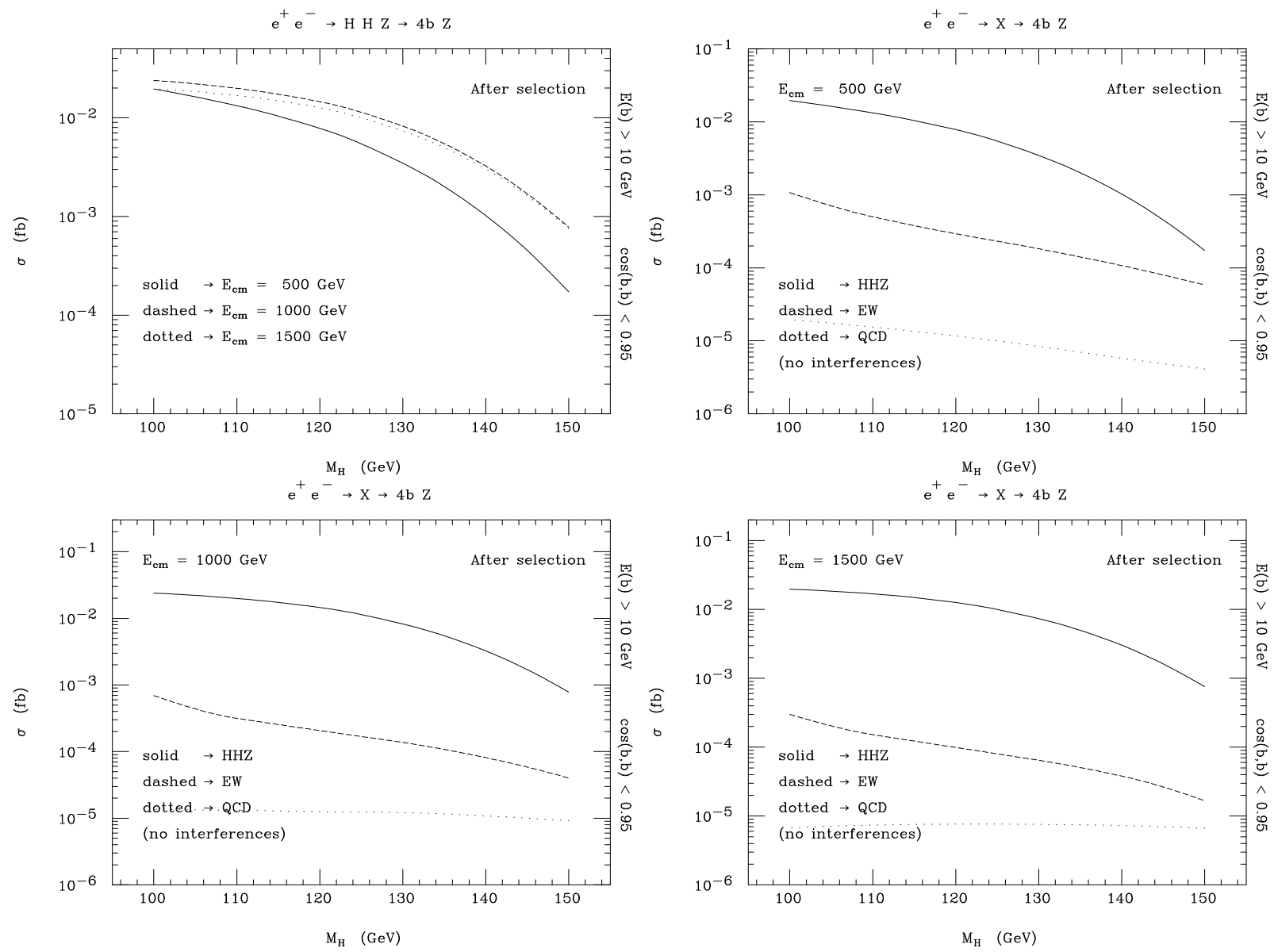

Figure 11: Top-left: cross sections in femtobarns for the signal at three different collider energies: 500, 1000 and $1500 \mathrm{GeV}$. Top-right(Bottom-left)[Bottom-right]: cross sections in femtobarns for the signal versus the EW and QCD backgrounds at 500(1000)[1500] GeV. Our acceptance cuts in energy and separation of the four $b$ quarks have been implemented along with the selection cuts of eq. (田). 